\title{
ESTUDO COMPARATIVO DA FORÇA DO OSCILADOR E DOS PARÂMETROS ÓPTICOS DE LIGAÇÃO NOS COMPOSTOS Nd(TFMS)3.9H2O, NdCl3.7H2O, NdNTA.3H2O e Nd(NO)3.5H2O EM SOLUÇÃO E NO ESTADO SÓLIDO
}

\section{C. M. BRAGA ${ }^{1}$; F. J. S. LIMA ${ }^{2}$; J. A. S. GRILO JUNIOR ${ }^{3}$; J.K.S. OLIVEIRA ${ }^{4}$}

Instituto Federal de Educação, Ciência e Tecnologia do Rio Grande do Norte ${ }^{1,3,4}$, Universidade Federal do Rio Grande do Norte ${ }^{2}$ jose.junior@ifrn.edu.br ${ }^{3}$

Artigo submetido em 28/11/2015 e aceito em 24/06/2019

DOI: $10.15628 /$ holos.2019.3780

\section{RESUMO}

O presente artigo tem por objetivo fazer uma comparação da força do oscilador e dos parâmetros ópticos de ligação nos compostos $\mathrm{Nd}(\mathrm{TFMS})_{3} .9 \mathrm{H}_{2} \mathrm{O}$, $\mathrm{NdCl}_{3} .7 \mathrm{H}_{2} \mathrm{O}, \mathrm{NdNTA} .3 \mathrm{H}_{2} \mathrm{O}$ e $\mathrm{Nd}\left(\mathrm{NO}_{3}\right)_{3} .5 \mathrm{H}_{2} \mathrm{O}$ no estado sólido. Para isso, foram estudados a influência dos grupos ligantes nos resultados obtidos para os parâmetros ópticos de ligação e força do oscilador. Verificamos a correlação entre o aumento ou a diminuição do envolvimento dos orbitais de fronteira com a polarizabilidade estática. Evidenciamos a importância do índice de refração experimental na avaliação da intensidade da transição. Comparamos os resultados obtidos para estado sólido com aqueles encontrados em sistemas em solução anteriormente estudados. De maneira geral, o trabalho pretende mostrar, que uma modificação do sistema, mais especificamente, no ambiente químico ao redor $\operatorname{Ln}^{3+}$ pode ser expressa a partir de valores de parâmetros ópticos de ligação, força do oscilador e polarizabilidades eletrônicas.

PALAVRAS-CHAVE: parâmetros ópticos, ligantes, estado sólido, força do oscilador.

\section{COMPARATIVE STUDY OF COMPOUNDS OF BOND PARAMETERS OPTICAL $\mathrm{Nd}(\mathrm{TFMS})_{3} .9 \mathrm{H}_{2} \mathrm{O}, \mathrm{NdCl}_{3} .7 \mathrm{H}_{2} \mathrm{O}, \mathrm{NdNTA} .3 \mathrm{H}_{2} \mathrm{O}$ e $\mathrm{Nd}(\mathrm{NO})_{3} .5 \mathrm{H}_{2} \mathrm{O}$ SOLUTION AND SOLID STATE}

At first, for compounds $\mathrm{Nd}(\mathrm{TFMS})_{3} .9 \mathrm{H}_{2} \mathrm{O}, \mathrm{NdCl}_{3} .7 \mathrm{H}_{2} \mathrm{O}$, NdNTA. $3 \mathrm{H}_{2} \mathrm{O}$ and $\mathrm{Nd}(\mathrm{NO} 3)_{3} .5 \mathrm{H}_{2} \mathrm{O}$ solid state, we studied the influence of ligand groups on the results obtained for the optical parameters of connection and strength oscillator. We verify the correlation between the increase or decrease in the involvement of frontier orbitals with static polarizability. We noted the importance of experimental refractive index in assessing the intensity of the transition. We compare the results obtained for solid state to those found in previously studied systems in solution. In general, the work aims to show that a system modification, more specifically, the chemical environment around $\mathrm{Ln}^{3+}$ can be expressed from connection of optical parameter values, the oscillator strength and electronic polarizabilities.

KEYWORDS: bond of parameters, ligands, solid state, oscillator strength. 


\section{INTRODUÇÃO}

Os 15 elementos do bloco $f$, lantanídeos, variando de lantânio $(Z=57)$ a lutécio $(Z=71)$, apresentam propriedades químicas semelhantes, devido aos orbitais $4 f$ internos. Esses orbitais exercem uma blindagem sobre os demais elétrons, originando um decréscimo gradual nos raios iônicos, decrescendo de 1,03 ̊̊ para o La ${ }^{3+}$ e 0,86 Å para o Lu+ (NI et al., 2015; LIMA, 1993).

A interação da radiação eletromagnética com a matéria causa vários fenômenos, entre eles, alterações nos níveis de energia dos orbitais e deformação de nuvens eletrônicas. O mecanismo de reorganização eletrônica dos íons quando há interação da radiação eletromagnética com um composto, é um processo bastante complicado e depende de suas energias de estabilização determinadas pelo campo ligante. O estudo da deformação de sua nuvem eletrônica em um determinado ambiente químico, quando interage com a radiação, pode ser útil na escolha dos grupos ligantes na síntese de um determinado complexo, objetivando uma maximização de suas propriedades ópticas e eletrônicas (LIMA et al, 2008).

A investigação de compostos por espectroscopia tem sido utilizada para elucidar diversos aspectos relativos a estabilidade, estrutura e reatividade de compostos. Alguns trabalhos com compostos de coordenação têm sido realizados e vasta é a sua literatura. Uma boa quantidade desses tem sido direcionada para a pesquisa de novos materiais usados em nanotecnologia de arquitetura molecular, e especialmente na área de compostos usados na interconversão da radiação bem como na conversão de luz em eletricidade em alguns dispositivos eletrônicos (LIMA et al; 2010).

As aplicações de íons $\mathrm{Ln}^{3+}$ estende-se por todos os campos importantes da alta tecnologia (SOM; KARMAKAR, 2011). Desde a década de 1960, compostos de coordenação com íons lantanídeos têm sido investigados pelos grupos de pesquisa em todo o mundo. $O$ interesse nesta classe de compostos tem atendido a diversos campos de atividades humanas, iniciando com a investigação acadêmica, passando pela descoberta e aplicação de novas técnicas de perscruta, e culminando com a confecção de novos materiais, objetivando sempre, aplicações em novas tecnologias. Os elementos lantanídeos têm se prestado como uma alternativa viável e estratégica na substituição de outros metais em determinados compostos, objetivando a melhoria da qualidade dos materiais, aumentando assim suas eficiências em certas propriedades físico-químicas peculiares. São conhecidas suas aplicações mais difundidas tais como fabricação de vidros especiais absorvedores, emissores e conversores de radiação uv visível, confecção de componentes eletroeletrônicos aplicados em displays LCD (liquid crystal display) e RCT (ray catode tube), síntese de substâncias geradoras de cores primárias em monitores de vídeo, manufatura de materiais magnéticos e supercondutores, entre outros. Toda esta diversidade de áreas de aplicação que envolve alta tecnologia se deve as suas características físicas e químicas peculiares, não observadas e em certos aspectos indisponíveis, em outros elementos (LIRA; LIMA; SILVA, 2004; SEREDIN; DAI, 2012; NATALI et al., 2013). Existem várias classificações desses elementos dos pontos de vista geoquímico e econômico. A classificação geoquímica, é dividida em grupos leve ( $\mathrm{La}, \mathrm{Ce}, \mathrm{Pr}, \mathrm{Nd}$, e Sm), médios (Eu, Gd, Tb, Dy, e Y) e pesados (Ho, Er, Tm, Yb, e Lu) (SEREDIN; DAI, 2012). 
As fórmulas estruturais dos ânions trifluorometanossulfonato, nitrilotriacetato e nitrato utilizados como ligantes, neste trabalho, foram construídas através do programa WebLab ViewerPro, conforme demonstradas nas figuras 1, 2 e 3. A Tabela 01 mostra as configurações eletrônicas, os estados de oxidação alcançados originando os respectivos termos espectroscópicos.

Tabela 01 - Configurações eletrônicas, estados de oxidação e seus respectivos termos espectroscópicos (BRAGA, 2004).

\begin{tabular}{|c|c|c|c|c|c|}
\hline \multirow{2}{*}{ Elemento } & \multirow{2}{*}{ Z } & \multicolumn{4}{|c|}{$\begin{array}{l}\text { Configuração eletrônica } \\
\text { (Termos espectroscópicos) }\end{array}$} \\
\hline & & $\operatorname{Ln}^{0}$ & $\mathrm{Ln}^{2+}$ & $\operatorname{Ln}^{3+}$ & $\operatorname{Ln}^{4+}$ \\
\hline La & 57 & $\begin{array}{c}{[\mathrm{Xe}] 5 d^{1} 6 s^{2}} \\
\left({ }^{2} D_{3 / 2}\right)\end{array}$ & & $\begin{array}{c}{[\mathrm{Xe}] 4 \mathrm{f}^{0}} \\
\left({ }^{1} \mathrm{~S}_{0}\right)\end{array}$ & \\
\hline $\mathrm{Ce}$ & 58 & $\begin{array}{c}{[\mathrm{Xe}] 4 \mathrm{f}^{1} 5 \mathrm{~d}^{1} 6 \mathrm{~s}^{2}} \\
\left({ }^{1} \mathrm{G}_{4}\right)\end{array}$ & & $\begin{array}{l}{[\mathrm{Xe}] 4 \mathrm{f}^{1}} \\
\left({ }^{2} \mathrm{~F}_{5 / 2}\right) \\
\end{array}$ & $\begin{array}{c}{[\mathrm{Xe}] 4 \mathrm{f}^{0}} \\
\left({ }^{1} \mathrm{~S}_{0}\right)\end{array}$ \\
\hline $\operatorname{Pr}$ & 59 & $\begin{array}{c}{[\mathrm{Xe}] 4 \mathrm{f}^{3} 6 \mathrm{~s}^{2}} \\
\left({ }^{4} \mathrm{I}_{g / 2}\right)\end{array}$ & & $\begin{array}{c}{[\mathrm{Xe}] 4 \mathrm{f}^{2}} \\
\left({ }^{3} \mathrm{H}_{4}\right)\end{array}$ & $\begin{array}{l}{[\mathrm{Xe}] 4 \mathrm{f}^{1}} \\
\left({ }^{2} \mathrm{~F}_{5 / 2}\right)\end{array}$ \\
\hline $\mathrm{Nd}$ & 60 & $\begin{array}{c}{[\mathrm{Xe}] 4 \mathrm{f}^{4} 6 \mathrm{~s}^{2}} \\
\left(\mathrm{I}_{4}\right)\end{array}$ & & $\begin{array}{c}{[\mathrm{Xe}] 4 \mathrm{f}^{3}} \\
\left({ }^{4} \mathrm{l} / \mathrm{g} / 2\right) \\
\end{array}$ & \\
\hline $\mathrm{Pm}$ & 61 & $\begin{array}{c}{[\mathrm{Xe}] 4 f^{5} 6 \mathrm{~s}^{2}} \\
\left({ }^{6} \mathrm{H}_{5 / 2}\right)\end{array}$ & & $\begin{array}{c}{[X e] 4 f^{4}} \\
(5 / 4)\end{array}$ & \\
\hline Sm & 62 & $\begin{array}{c}{[\mathrm{Xe}] 4 f^{6} 6 s^{2}} \\
\left({ }^{7} \mathrm{~F}_{0}\right)\end{array}$ & $\begin{array}{c}{[\mathrm{Xe}] 4 \mathrm{f}^{6}} \\
\left({ }^{7} \mathrm{~F}_{0}\right) \\
\end{array}$ & $\begin{array}{c}{[\mathrm{Xe}] 4 \mathrm{f}^{5}} \\
\left({ }^{6} \mathrm{H}_{5 / 2}\right) \\
\end{array}$ & \\
\hline Eu & 63 & $\begin{array}{c}{[\mathrm{Xe}] 4 \mathrm{f}^{7} 6 \mathrm{~s}^{2}} \\
\left({ }^{8} \mathrm{~S}_{7 / 2}\right)\end{array}$ & $\begin{array}{c}{[\mathrm{Xe}] 4 \mathrm{f}^{7}} \\
\left({ }^{8} \mathrm{~S}_{7 / 2}\right) \\
\end{array}$ & $\begin{array}{c}{[\mathrm{Xe}] 4 f^{6}} \\
\left({ }^{7} \mathrm{~F}_{0}\right)\end{array}$ & \\
\hline $\mathrm{Gd}$ & 64 & $\begin{array}{c}{[\mathrm{Xe}] 4 \mathrm{f}^{7} 5 \mathrm{~d}^{1} 6 \mathrm{~s}^{2}} \\
\left({ }^{9} \mathrm{D}_{2}\right)\end{array}$ & & $\begin{array}{c}{[\mathrm{Xe}] 4 \mathrm{f}^{7}} \\
\left({ }^{8} \mathrm{~S}_{7 / 2}\right)\end{array}$ & \\
\hline $\mathrm{Tb}$ & 65 & $\begin{array}{c}{[\mathrm{Xe}] 4 \mathrm{f}^{9} 6 \mathrm{~s}^{2}} \\
\left({ }^{6} \mathrm{H}_{15 / 2}\right)\end{array}$ & & $\begin{array}{c}{[\mathrm{Xe}] 4 f^{8}} \\
\left({ }^{7} \mathrm{~F}_{6}\right)\end{array}$ & $\begin{array}{r}{[\mathrm{Xe}] 4 \mathrm{f}^{7}} \\
\left({ }^{8} \mathrm{~S}_{7 / 2}\right)\end{array}$ \\
\hline Dy & 66 & $\begin{array}{c}{[\mathrm{Xe}] 4 \mathrm{f}^{10} 6 \mathrm{~s}^{2}} \\
\left({ }^{5} / 8\right)\end{array}$ & & $\begin{array}{l}{[\mathrm{Xe}] 4 \mathrm{f}^{9}} \\
\left({ }^{6} \mathrm{H}_{15 / 2}\right)\end{array}$ & \\
\hline Ho & 67 & $\begin{array}{c}{[\mathrm{Xe}] 4 \mathrm{f}^{11} 6 \mathrm{~s}^{2}} \\
\left({ }^{4} I_{15 / 2}\right)\end{array}$ & & $\begin{array}{c}{[\mathrm{Xe}] 4 \mathrm{f}^{10}} \\
\left({ }^{5} 1_{8}\right)\end{array}$ & \\
\hline $\mathrm{Er}$ & 68 & $\begin{array}{c}{[\mathrm{Xe}] 4 \mathrm{f}^{12} 6 \mathrm{~s}^{2}} \\
\left({ }^{3} \mathrm{H}_{6}\right)\end{array}$ & & $\begin{array}{c}{[\mathrm{Xe}] 4 \mathrm{f}^{11}} \\
\left(\mathrm{I}_{15 / 2}\right)\end{array}$ & \\
\hline $\mathrm{Tm}$ & 69 & $\begin{array}{c}{[\mathrm{Xe}] 4 \mathrm{f}^{13} 6 \mathrm{~s}^{2}} \\
\left({ }^{2} \mathrm{~F}_{7 / 2}\right)\end{array}$ & & $\begin{array}{c}{[\mathrm{Xe}] 4 \mathrm{f}^{12}} \\
\left({ }^{3} \mathrm{H}_{6}\right)\end{array}$ & \\
\hline $\mathrm{Yb}$ & 70 & $\begin{array}{c}{[\mathrm{Xe}] 4 \mathrm{f}^{14} 6 \mathrm{~s}^{2}} \\
\left({ }^{1} \mathrm{~S}_{0}\right)\end{array}$ & $\begin{array}{c}{[\mathrm{Xe}] 4 \mathrm{f}^{14}} \\
\left({ }^{1} \mathrm{~S}_{0}\right)\end{array}$ & $\begin{array}{c}{[\mathrm{Xe}] 4 \mathrm{f}^{13}} \\
\left({ }^{2} \mathrm{~F}_{7 / 2}\right)\end{array}$ & \\
\hline Lu & 71 & $\begin{array}{c}{[X e] 4 f^{14} 5 d^{1} 6 s^{2}} \\
\left({ }^{3} D_{3 / 2}\right)\end{array}$ & & $\begin{array}{c}{[\mathrm{Xe}] 4 \mathrm{f}^{14}} \\
\left({ }^{1} \mathrm{~S}_{0}\right)\end{array}$ & \\
\hline
\end{tabular}




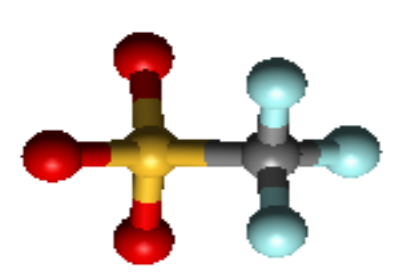

Figura 1: Modelo estrutural para o ânion trifluorometanossulfonato $\left(\mathrm{TFMS}=\mathrm{CF}_{3} \mathrm{SO}_{3}^{-}\right)$.

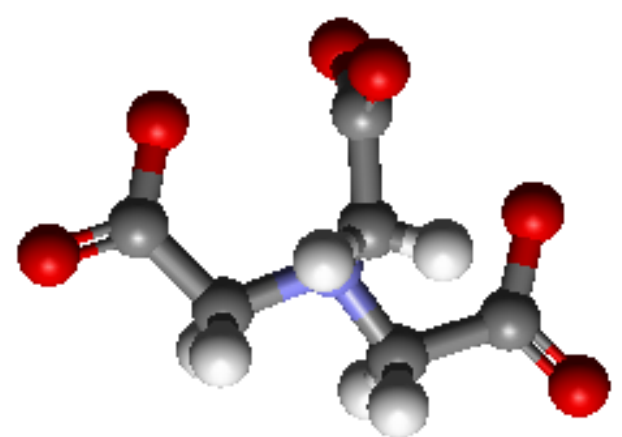

Figura 2: Modelo estrutura do íon nitrilotriacetato $\left(N T A=\mathrm{C}_{6} \mathrm{O}_{6} \mathrm{~N}^{3-}\right)$.

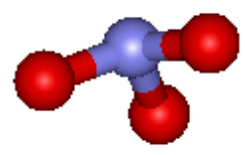

Figura 3: Modelo estrutura do íon nitrato $\left(\mathrm{NO}_{3}^{-}\right)$. 


\section{Legenda para os átomos contidos nas fórmulas estruturais:}

Azul claro = flúor.

Azul escuro = nitrogênio.

Cinza = hidrogênio.

Dourado = enxofre.

Grafite = carbono.

Verde $=$ cloro .

Vermelho = oxigênio.

\section{REVISÃO BIBLIOGRÁFICA}

\subsection{Parâmetro Nefelauxético $(\beta)$}

O termo efeito nefelauxético, vem do grego, e foi originalmente proposto por Christian Klixbull Jorgensen. Os estudos realizados por Jorgensen revelaram que o efeito nefelauxético, expressa uma razão entre o parâmetro Racah, devido à interação do íon central com os ligantes em relação ao seu valor no íon livre contido em um composto considerado padrão (SUCHOCKI; BIERNACKI; GRINBERG, 2007). Os parâmetros de repulsão intereletrônica são determinados experimentalmente (ANTHON; SCHAFFER, 2002) e o fato do valor deste parâmetro ser menor nos complexos e com tendência em decrescer com o aumento da intensidade de campo produzida pelos ligantes reflete a natureza da ligação química (GUSHIKEM, 2005). As integrais $\left\langle r^{k}\right\rangle_{4 f}$, (com k = 2, 4, 6) desempenham papel crucial na determinação dos parâmetros de campo cristalino de íons Ln3+ em sólidos (ANGELOV, 2004).

A equação 01 representa o parâmetro nefelauxético a partir do parâmetro de Racah.

$$
\beta=\frac{B_{\text {comp }}}{B_{\text {ion }}}
$$

onde:

$B_{\text {comp }}=$ parâmetro de Racah referente a interação do íon com o ligante.

$B_{\text {íon }}=$ parâmetro de Racah referente ao íon livre (composto considerado padrão).

A partir de modificações realizadas na equação 01 obtêm-se a equação 02 .

$$
\beta=\frac{\bar{v}_{\text {comp }}}{\bar{v}_{\text {ion }}}
$$

onde: 


$$
\begin{aligned}
& \bar{v}_{c o m p}=\text { número de onda do baricentro da transição do composto estudado. } \\
& \bar{v}_{i o n}=\text { número de onda do baricentro da transição do íon no padrão. }
\end{aligned}
$$

\subsection{Parâmetro de Covalência de Sinha $(\delta)$}

O estudo das propriedades espectroscópicas e a avaliação dos parâmetros ópticoseletrônicos de complexos contendo íons lantanídeos tem sido largamente investigado na literatura devido às diversas possibilidades de aplicações, como por exemplo em lasers, sensores luminescentes para espécies químicas, dosímetros UV, etc. O estudo da polarizabilidade eletrônica pode ser aplicado no acompanhamento dos estudos eletrônicos dos estados intermediários de transição, em processos fotoquímicos e em reações químicas fotocatalisadas de interesse na obtenção de substâncias estereoseletivas. Um estudo detalhado dos mecanismos de excitação de moléculas pode ser útil no acompanhamento dos fenômenos de obtenção e controle de espécies no estado do complexo ativado, através da avaliação da deformação da nuvem eletrônica das espécies opticamente ativas (PEDROSA et al, 2004).

O comportamento dos orbitais no que se refere a covalência entre lantanídeos e grupos ligantes é avaliado considerando-se o efeito nefelauxético através do parâmetro de Sinha, o qual é definido pela equação 03 .

$$
\delta=\frac{1-\bar{\beta}}{\bar{\beta}} \times 100 \%
$$

Em uma avaliação dos resultados encontra-se um intervalo para indicar uma tendência no que se refere ao caráter covalente:

se $\delta<1,5 \%$, caráter covalente pouco significativo;

se $\delta>1,5 \%$, caráter covalente significativo.

\subsection{Fator de Covalência $\left(b^{1 / 2}\right)$}

A interação metal-ligante envolvendo compostos contendo íons $\mathrm{Ln}^{3+}$ é predominantemente eletrostática. Portanto, desde 1950, sabe-se que o caráter covalente é pequeno nesses compostos. A forte ionicidade nas ligações contendo esses íons se reflete na ausência de variação significativa nos efeitos de campo ligante em seus complexos e na correlação das tendências na termodinâmica e cinética de complexação. Outra consequência do caráter iônico da ligação, nos complexos, é um elevado números de coordenação (3 a 12) e geometrias que são devidas a fatores estereoquímicos e eletrostáticos (CHOPPIN, 2002).

O fator de covalência, $b^{1 / 2}$, indica a extensão do caráter covalente da ligação, avaliando quantitativamente o envolvimento dos orbitais $4 \mathrm{f}$ dos lantanídeos com os orbitais dos grupos ligantes. 
$b^{1 / 2}=\left[\frac{1}{2}(1-\bar{\beta})\right]^{1 / 2}$

\subsection{Força do Oscilador}

A intensidade de uma transição eletrônica pode ser mensurada através da força do oscilador, a qual é proporcional a área sob a curva dos espectros de absorção. As intensidades das transições eletrônicas são fortemente dependentes do ambiente do íon (NISSAMUDEEN; GOPCHANDRAN, 2010). A força do oscilador experimental é calculada a partir do espectro de absorção(LIU et al., 2008), conforme equação 05. A força do oscilador, $P_{0 K}$, é definida como sendo a relação entre a probabilidade de uma transição experimental em relação ao padrão. Portanto, essa propriedade é adimensional, pois é a razão entre duas probabilidades absolutas (LIMA et al, 1997).

$P_{0 K}=\frac{m_{e} \cdot c^{2}}{\pi \cdot e^{2} \cdot N} \cdot \int \varepsilon(\bar{v}) \cdot d(\bar{v})_{\exp }$

onde:

$m_{e}=$ massa do elétron.

$c=$ velocidade daluz.

$e=$ carga do elétron.

$\varepsilon=$ absortividade molar .

$N=$ número de Avogadro.

$P_{0 K}=\frac{\int \varepsilon(\bar{v}) \cdot d\left(\bar{v}_{\exp }\right.}{\int \varepsilon(\bar{v}) \cdot d(\bar{v})_{r e f}}=4,319 \cdot 10^{-9} \int \varepsilon(\bar{v}) \cdot d(\bar{v})_{\exp }$

$P_{0 K}=4,319 \cdot 10^{-9} \frac{9 n}{\left(n^{2}+2\right)^{2}} \int \varepsilon(\bar{v}) \cdot d(\bar{v})_{\exp }$

onde:

$n=$ índice derefração

\section{METODOLOGIA}

As transições estudadas foram ${ }^{4} I_{9 / 2} \rightarrow{ }^{4} G_{5 / 2},{ }^{2} G_{7 / 2}$ para os compostos $\mathrm{Nd}(\mathrm{TFMS})_{3} .9 \mathrm{H}_{2} \mathrm{O}$ (intervalo espectral $=560,1-600 \mathrm{~nm}$ ), $\mathrm{NdCl}_{3} .7 \mathrm{H}_{2} \mathrm{O}$ (intervalo espectral $=560-600 \mathrm{~nm}$ ), NdNTA.3 $\mathrm{H}_{2} \mathrm{O}$, 
$\mathrm{NTA}=$ nitrilotriacetato $\mathrm{C}_{6} \mathrm{O}_{6} \mathrm{~N}^{3-}$ (intervalo espectral $=560,1-600 \mathrm{~nm}$ ) e $\mathrm{Nd}\left(\mathrm{NO}_{3}\right)_{3} .5 \mathrm{H}_{2} \mathrm{O}$ (intervalo espectral $=560-590 \mathrm{~nm}$ ), e a ${ }^{4} I_{9 / 2} \rightarrow{ }^{4} G_{7 / 2},{ }^{2} G_{9 / 2}$ para os compostos Nd(TFMS)3.9H20 (intervalo espectral $=500-530 \mathrm{~nm}$ ), e NdCl3.7H2O (intervalo espectral $=560-600 \mathrm{~nm}$ ). 0 estudo foi direcionado para a primeira transição do $\mathrm{Nd3}+$, a qual é hipersensitiva, ou seja, é modificada a partir de uma nova conformação química adquirida pelo sistema. Os compostos, no estado sólido, formaram uma dispersão onde o glicerol da marca sigma foi utilizado como dispersante. Os espectros foram registrados em um espectrofotômetro uv-vis HITACHI de duplo feixe com precisão de comprimento de onda de $\pm 0,1 \mathrm{~nm}$ e absorbância $\pm 0,002$. A força do oscilador foi calculada a partir do programa SIMP2FOSC escrito em QBASIC. Esse programa utiliza a regra de Simpson para o cálculo de áreas irregulares.

\section{RESULTADOS E DISCUSSÕES}

Os resultados da Tabela 2 obtidos para os parâmetros espectroscópicos de ligação indicam uma ionicidade elevada dos compostos resultado de um deslocamento do baricentro do número de onda no sentido de maior energia em relação ao padrão, sugerindo que o envolvimento dos orbitais do $\mathrm{Nd}^{3+}$ com os grupos ligantes é mínimo. Essa característica é mais acentuada no $\mathrm{NdNTA} \cdot 3 \mathrm{H}_{2} \mathrm{O}$ evidenciando a influência do ânion. Outro fator que deve ser destacado é a correção realizada para o índice de refração, pois considerando o índice ideal $\left(n_{\text {ideal }}\right)$ implica em afirmar que a velocidade de propagação da radiação eletromagnética, ao interagir com o sistema, não sofre alteração, o que é um equívoco. Porém, introduzindo o índice de refração experimental $\left(n_{\exp }\right)$ nas equações, admitese uma diminuição da velocidade da radiação ao interagir com o meio. Dessa maneira, verificou-se que essa correção influenciou a intensidade da transição, pois para todos os compostos, os valores de $\mathrm{P}_{0 \mathrm{k}}$ admitindo $\mathrm{n}_{\text {ideal }}$ são aproximadamente $23 \%$ maiores do que aqueles a partir de $\mathrm{n}_{\exp }$. Analisando ainda, pode-se dizer que sem a correção admite-se que as perdas são mínimas, ou seja, a energia seria quase totalmente transferida para a ocorrência do salto quântico. Porém, a rede cristalina de cada material interage de maneira específica com os fótons, originando dessa forma uma diminuição da velocidade da luz bem como absorvendo parte da energia.

Na Tabela 3 encontram-se reunidos resultados para os parâmetros ópticos de ligação para alguns compostos em solução. Comparando os dados contidos na Tabela $1 \mathrm{com}$ os da Tabela 2 resulta a Tabela 3, a qual apresenta uma comparação entre os valores dos parâmetros e força do oscilador no estado sólido e em solução para a transição ${ }^{4} I_{9 / 2} \rightarrow{ }^{4} G_{5 / 2},{ }^{2} G_{7 / 2}$.

Analisando as informações contidas na Tabela 4, observa-se que o composto $\mathrm{Nd}(\mathrm{TFMS})_{3} .9 \mathrm{H}_{2} \mathrm{O}$ em metanol apresenta a maior covalência. A ionicidade mais elevada e a maior probabilidade de transição é verificada em acetonitrila. Para o composto $\mathrm{NdCl}_{3} .7 \mathrm{H}_{2} \mathrm{O}$ o menor envolvimento entre os orbitais do íon $\mathrm{Nd}^{3+}$ com os dos ligantes se deu no estado sólido. A covalência mais elevada observou-se em etanol, bem como a maior probabilidade de transição. Portanto, fica caracterizado que alterações na vizinhança do íon $\mathrm{Nd}^{3+}$, mais especificamente as contribuições do 
solvente, influenciaram os resultados. A legenda para todas as tabelas é: $\mathrm{S}=$ solvente; $\mathrm{A}=$ acetonitrila; $\mathrm{M}=$ metanol; $\mathrm{E}$ = etanol.

As figuras de 1 a 4 mostram espectros de absorção no estado sólido para as espécies estudadas a uma temperatura de $25^{\circ} \mathrm{C}$. As áreas sob a curva desses espectros foram utilizadas para o cálculo da força do oscilador.

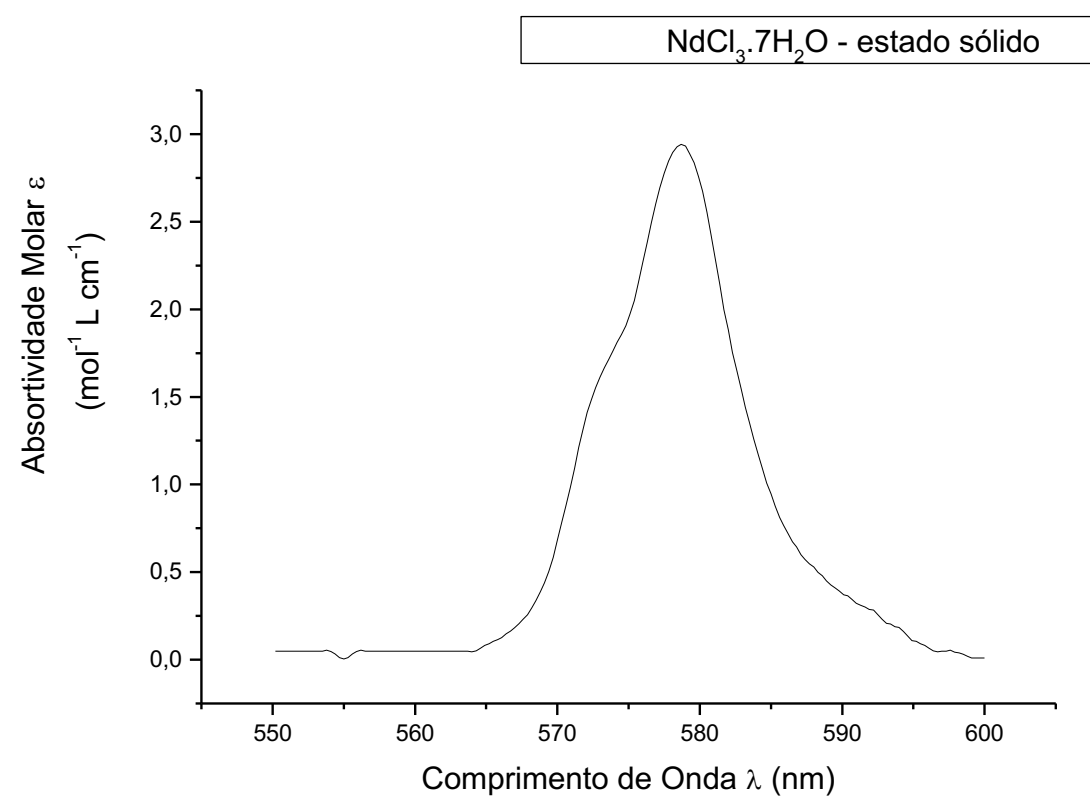

Figura 1: Espectro de absorção para a transição ${ }^{4} I_{9 / 2} \rightarrow{ }^{4} G_{5 / 2},{ }^{2} G_{7 / 2}$.

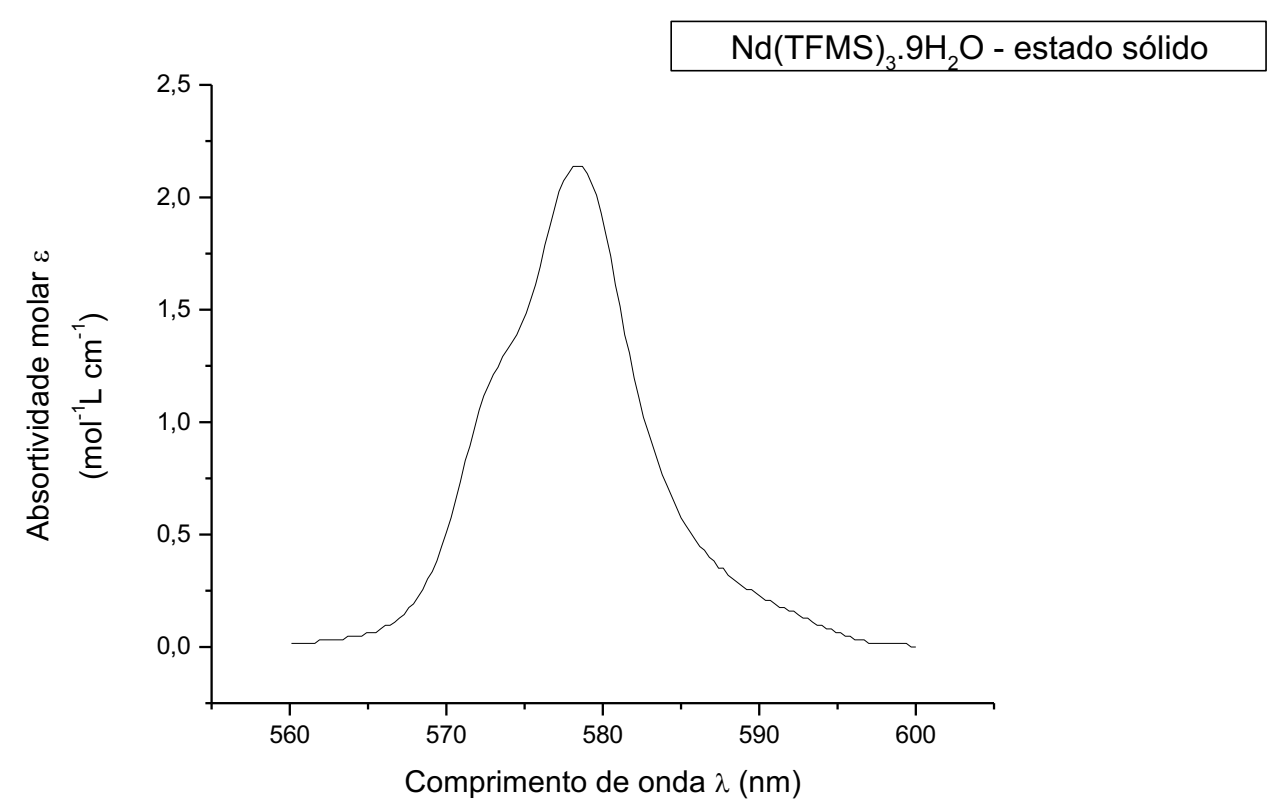


Figura 2: Espectro de absorção para a transição ${ }^{4} I_{9 / 2} \rightarrow{ }^{4} G_{5 / 2},{ }^{2} G_{7 / 2}$.

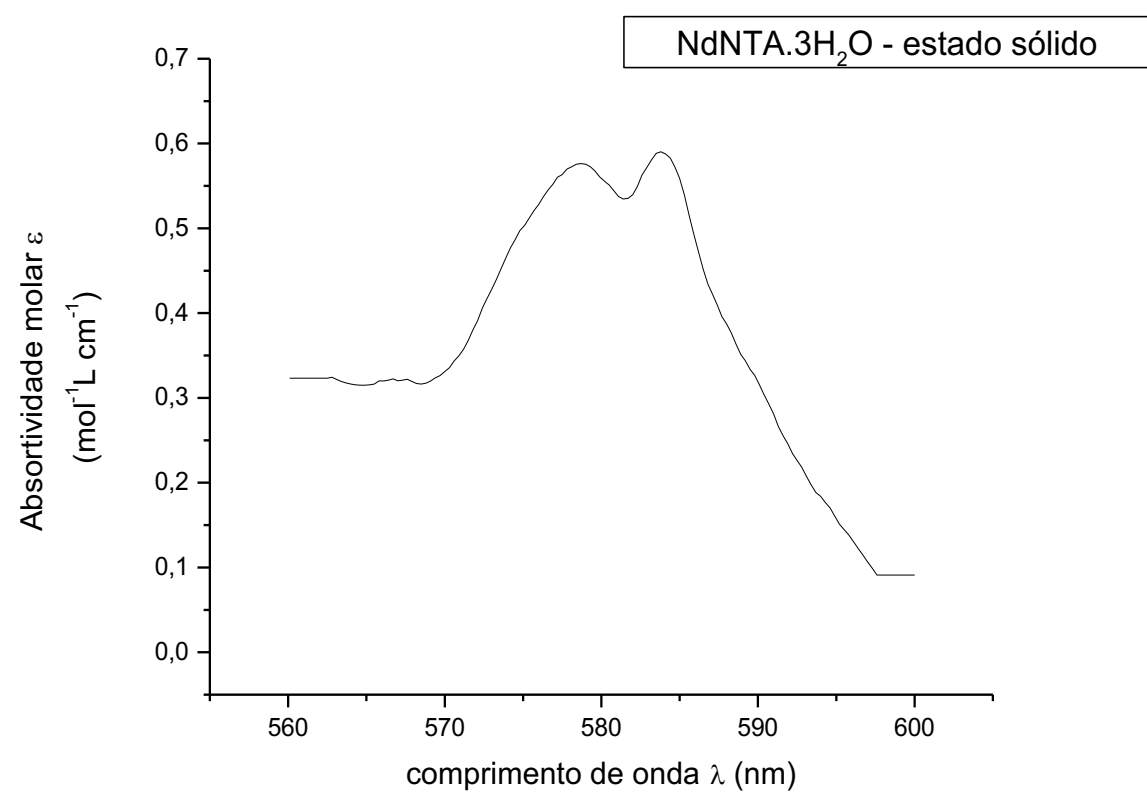

Figura 3: Espectro de absorção para a transição ${ }^{4} I_{9 / 2} \rightarrow{ }^{4} G_{5 / 2},{ }^{2} G_{7 / 2}$.

$\mathrm{Nd}\left(\mathrm{NO}_{3}\right)_{3} \cdot 5 \mathrm{H}_{2} \mathrm{O}$ - estado sólido

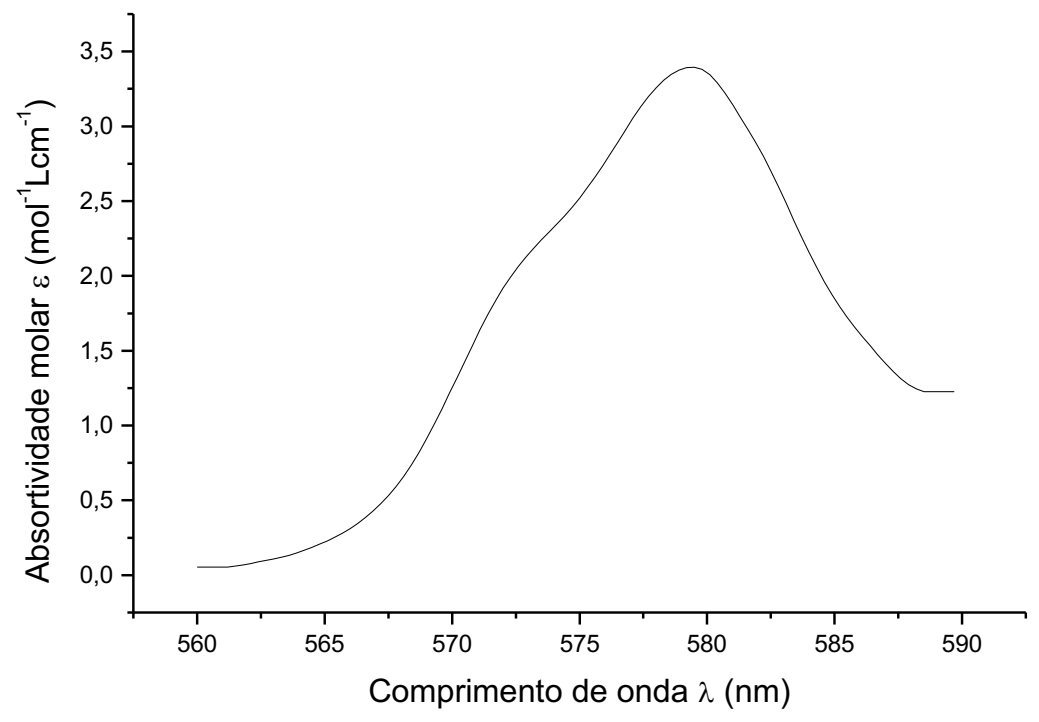




$$
\text { Figura 4: Espectro de absorção para a transição }{ }^{4} I_{9 / 2} \rightarrow{ }^{4} G_{5 / 2},{ }^{2} G_{7 / 2} \text {. }
$$

Tabela 2: Índice de refração $(n)$, baricentro do número de onda $(\bar{v})$, parâmetro nefelauxético $(\bar{\beta})$, parâmetro de covalência de Sinha $(\delta)$, fator de covalência $\left(b^{1 / 2}\right)$ e força do oscilador $\left(P_{0 k}\right)$, no estado sólido.

\begin{tabular}{|c|c|c|c|c|c|c|c|c|}
\hline \multirow{2}{*}{ Composto } & \multicolumn{2}{|c|}{$n$} & \multirow{2}{*}{$\bar{v}\left(\mathrm{~cm}^{-1}\right)$} & \multirow{2}{*}{$\bar{\beta}$} & \multirow{2}{*}{$\delta(\%)$} & \multirow{2}{*}{$b^{1 / 2}$} & \multicolumn{2}{|c|}{$P_{0 k} \cdot 10^{-6}$} \\
\hline & $n_{\text {ideal }}$ & $n_{\text {exp }}$ & & & & & $n_{\text {ideal }}$ & $n_{\text {exp }}$ \\
\hline $\begin{array}{c}\mathrm{Nd}(\mathrm{TFMS})_{3} .9 \mathrm{H}_{2} \mathrm{O} \\
{ }^{4} I_{9 / 2} \rightarrow{ }^{4} G_{5 / 2},{ }^{2} G_{7 / 2} \\
{ }^{4} I_{9 / 2} \rightarrow{ }^{4} G_{7 / 2},{ }^{2} G_{9 / 2}\end{array}$ & 1,000 & 1,459 & $\begin{array}{l}17292 \\
19330\end{array}$ & $\begin{array}{c}0,9979 \\
-\end{array}$ & $\begin{array}{c}0,2140 \\
-\end{array}$ & $\begin{array}{c}0,0327 \\
-\end{array}$ & $\begin{array}{l}3,147 \\
1,259\end{array}$ & $\begin{array}{l}2,424 \\
0,970\end{array}$ \\
\hline $\begin{array}{c}\mathrm{NdCl}_{3} .7 \mathrm{H}_{2} \mathrm{O} \\
{ }^{4} I_{9 / 2} \rightarrow{ }^{4} G_{5 / 2},{ }^{2} G_{7 / 2} \\
{ }^{4} I_{9 / 2} \rightarrow{ }^{4} G_{7 / 2},{ }^{2} G_{9 / 2}\end{array}$ & 1,000 & 1,465 & $\begin{array}{l}17289 \\
19403\end{array}$ & $\begin{array}{c}0,99602 \\
-\end{array}$ & $\begin{array}{c}0,400 \\
-\end{array}$ & $\begin{array}{c}0,0446 \\
-\end{array}$ & $\begin{array}{l}4,646 \\
2,868\end{array}$ & $\begin{array}{l}3,557 \\
2,196\end{array}$ \\
\hline $\begin{array}{c}\text { NdNTA.3 } \mathrm{H}_{2} \mathrm{O} \\
{ }^{4} I_{9 / 2} \rightarrow{ }^{4} G_{5 / 2},{ }^{2} G_{7 / 2}\end{array}$ & 1,000 & 1,467 & 17300 & 0,9983 & 0,1676 & 0,0289 & 1,860 & 1,425 \\
\hline $\begin{array}{c}\mathrm{Nd}\left(\mathrm{NO}_{3}\right)_{3} \cdot 5 \mathrm{H}_{2} \mathrm{O} \\
{ }^{4} I_{9 / 2} \rightarrow{ }^{4} G_{5 / 2},{ }^{2} G_{7 / 2}\end{array}$ & 1,000 & 1,466 & 17285 & 0,9975 & 0,2514 & 0,0354 & 6,1576 & 4,719 \\
\hline
\end{tabular}


Tabela 3: Parâmetros Ópticos de Ligação para os compostos $\mathrm{Nd}(\mathrm{TFMS})_{3} .9 \mathrm{H}_{2} \mathrm{O}, \mathrm{NdCl}_{3} .7 \mathrm{H}_{2} \mathrm{O}$ referente a transição ${ }^{4} I_{9 / 2} \rightarrow{ }^{4} G_{5 / 2},{ }^{2} G_{7 / 2}$ em solução (BATISTA, et al., 2002; SILVA, A. G. et al, 1996).

\begin{tabular}{|c|c|c|c|c|c|}
\hline Compostos & $S$ & $\bar{\beta}$ & $\delta(\%)$ & $b^{1 / 2}$ & $P_{0 K} \times 10^{-6}$ \\
\hline \multicolumn{6}{|l|}{$\mathrm{Nd}(\mathrm{TFMS})_{3} .9 \mathrm{H}_{2} \mathrm{O}$} \\
\hline${ }^{4} I_{9 / 2} \rightarrow{ }^{4} G_{5 / 2},{ }^{2} G_{7 / 2}$ & \multirow[t]{2}{*}{ A } & \multirow{2}{*}{$\begin{array}{l}0,998 \\
1,000\end{array}$} & 0,190 & 0,031 & 6,946 \\
\hline${ }^{4} I_{9 / 2} \rightarrow{ }^{4} G_{7 / 2},{ }^{2} G_{9 / 2}$ & & & - & - & 4,884 \\
\hline \multicolumn{6}{|l|}{$\mathrm{Nd}(\mathrm{TFMS})_{3} .9 \mathrm{H}_{2} \mathrm{O}$} \\
\hline${ }^{4} I_{9 / 2} \rightarrow{ }^{4} G_{5 / 2},{ }^{2} G_{7 / 2}$ & \multirow[t]{2}{*}{ M } & 0,995 & 0,533 & 0,052 & 5,532 \\
\hline${ }^{4} I_{9 / 2} \rightarrow{ }^{4} G_{7 / 2},{ }^{2} G_{9 / 2}$ & & 0,997 & 0,341 & 0,041 & 3,228 \\
\hline \multicolumn{6}{|l|}{$\mathrm{NdCl}_{3} .7 \mathrm{H}_{2} \mathrm{O}$} \\
\hline${ }^{4} I_{9 / 2} \rightarrow{ }^{4} G_{5 / 2},{ }^{2} G_{7 / 2}$ & \multirow[t]{2}{*}{ M } & 0,9894 & 1,0714 & 0,0728 & 13,441 \\
\hline${ }^{4} I_{9 / 2} \rightarrow{ }^{4} G_{7 / 2},{ }^{2} G_{9 / 2}$ & & 0,9954 & 0,4621 & 0,0480 & 6,426 \\
\hline \multicolumn{6}{|l|}{$\mathrm{NdCl}_{3} .7 \mathrm{H}_{2} \mathrm{O}$} \\
\hline${ }^{4} I_{9 / 2} \rightarrow{ }^{4} G_{5 / 2},{ }^{2} G_{7 / 2}$ & \multirow[t]{2}{*}{ E } & 0,9876 & 1,2556 & 0,0787 & 15,484 \\
\hline${ }^{4} I_{9 / 2} \rightarrow{ }^{4} G_{7 / 2},{ }^{2} G_{9 / 2}$ & & 0,9956 & 0,4419 & & 6,667 \\
\hline
\end{tabular}


Tabela 4: Comparação entre parâmetros ópticos de ligação e força do oscilador para os sistemas no estado sólido e em solução.

\begin{tabular}{c|c|c|c|c|c|c}
\hline \multirow{2}{*}{ Parâmetros } & \multicolumn{5}{|c}{ Compostos } \\
\cline { 2 - 7 } & \multicolumn{3}{|c}{$\mathrm{Nd}(\mathrm{TFMS})_{3.9 \mathrm{H}_{2} \mathrm{O}}$} & \multicolumn{3}{c}{$\mathrm{NdCl}_{3} .7 \mathrm{H}_{2} \mathrm{O}$} \\
\cline { 2 - 7 } & Sólido & $\mathrm{A}$ & $\mathrm{M}$ & Sólido & $\mathrm{M}$ & $\mathrm{E}$ \\
\cline { 2 - 7 } & 0,9979 & 0,998 & 0,995 & 0,99602 & 0,9894 & 0,9876 \\
\hline$\delta$ & 0,2140 & 0,190 & 0,533 & 0,4000 & 1,0714 & 1,2556 \\
\hline$b^{1 / 2}$ & 0,0327 & 0,031 & 0,052 & 0,0446 & 0,0728 & 0,0787 \\
\hline$P_{0 k} \times 10^{-6}$ & 2,424 & 6,946 & 5,532 & 3,557 & 13,441 & 15,484 \\
\hline
\end{tabular}

\section{CONCLUSÃO}

Os espectros para os compostos $\mathrm{Nd}(\mathrm{TFMS})_{3} .9 \mathrm{H}_{2} \mathrm{O}, \mathrm{NdCl}_{3} .7 \mathrm{H}_{2} \mathrm{O}, \mathrm{NdNTA} .3 \mathrm{H}_{2} \mathrm{O}$ e $\mathrm{Nd}\left(\mathrm{NO}_{3}\right)_{3} .5 \mathrm{H}_{2} \mathrm{O}$ no estado sólido referentes a transição ${ }^{4} I_{9 / 2} \rightarrow{ }^{4} G_{5 / 2},{ }^{2} G_{7 / 2}$ revelaram valores para os parâmetros ópticos de ligação que indicam uma pequena interação entre os orbitais do metal com os dos grupos ligantes. A menor interação foi observada para o composto $\mathrm{NdNTA} \cdot 3 \mathrm{H}_{2} \mathrm{O}$. A comparação dos resultados dos parâmetros espectroscópicos no estado sólido com aqueles em solução, indicou que a interação do solvente com o centro metálico proporcionou uma covalência mais elevada em solução do que no estado sólido.

Portanto, um estudo comparativo de sistemas no estado sólido e em solução mostra que as propriedades dos grupos ligantes assumem papel fundamental nos resultados obtidos. Os resultados desse estudo comparativo realizado para os sistemas em solução e no estado sólido convergem no sentido de que outros fatores que não sejam somente a interação metal-ligante e natureza do grupo ligante podem contribuir modificando o ambiente químico. 


\section{REFERÊNCIAS BIBLIOGRÁFICAS}

ANGELOV, B. M. (2004). Nephelauxetic effect and $\left\langle r^{k}\right\rangle_{4 f}$ radial integrals of $\mathrm{Pr}^{3+}$ in crystals. Journal of Alloys And Compounds, v. 384, p.76-79.

ANTHON, C.; SCHAFFER, C. E. (2002). Toward understanding nephelauxetism: interelectronic repulsion in gaseous dq ions computed by Kohn-Sham DFT. Coordination Chemistry Reviews, v. 226, p.17-38.

BATISTA, M. K. S. et al. (2002). Parâmetros cinéticos da degradação térmica de complexos entre percloratos de terras raras com lactama. Ecl. Quím., v.27, p.81-91.

BRAGA, C. C. M. (2004). Estudo, Interpretação e Comparação dos Parâmetros Ópticos de Ligação, Força do Oscilador e Polarizabilidades Eletrônicas dos Compostos NdNTA. $3 \mathrm{H}_{2} \mathrm{O}$, $\mathrm{Nd}(\mathrm{TFMS})_{3} .9 \mathrm{H}_{2} \mathrm{O}, \mathrm{NdCl}_{3} .7 \mathrm{H}_{2} \mathrm{O}$ e $\mathrm{Nd}\left(\mathrm{NO}_{3}\right)_{3} .5 \mathrm{H}_{2} \mathrm{O}$ em Solução e no Estado Sólido. 2004. $75 \mathrm{f}$. Dissertação (Mestrado) - Curso de Química, Departamento de Química, Universidade Federal do Rio Grande do Norte, Natal.

BURDICK, G.W.; ROBERTSON, R.D.; SUMMERSCALES, R.L. (2001). Electric-dipole $4 \mathrm{f}^{\mathrm{N}}-4 \mathrm{f}^{\mathrm{N}}$ transition intensity parametrizations for lanthanides: an examination of multiple local minima. Journal of Alloys And Compounds, 324(323), p.778-782.

CHOPPIN, Gregory R.. Covalency in f-element bonds. Journal of Alloys And Compounds, n. 344, p.5559, 2002.

GUSHIKEM, Y. (2005). Espectros eletrônicos de alguns complexos de geometria octaédrica de $\mathrm{Ni}^{2+}$ : uma introdução prática à teoria do campo cristalino no curso de graduação. Quimica Nova, 28(1), p.153-156.

LIMA, F. J. S. et al. (1997). Polarizabilidade Estática e dinâmica em Transições Eletrônicas de Compostos de Transição d e f, 37으 Cong. Assoc. Bras. de Quím.

LIMA, F. J. S. et al. (2008). Polarizabilidades eletrônicas de íons de terras raras em complexos de $\mathrm{Nd}(\mathrm{ClO} 4)_{3}(\mathrm{MMNO})_{6}$ e $\mathrm{Er}(\mathrm{ClO} 4)_{3}(\mathrm{MMNO})_{6}$ em solução. Tchê Química, Porto Alegre, v. 5, n. 9, p.38-43.

LIMA, F. J. S. et al. (2010). Parâmetros de ligação e força do oscilador dos maleatos de neodímio e érbio hidratados. Tchê Química, Porto Alegre, v. 7, n. 14, p.52-56.

LIRA, D. C. et al (2004). Síntese, caracterização e estudos térmicos, vibracionais e condutimétricos dos cloretos de praseodímio e neodímio com a 1,10 - fenantrolina. Tchê Química, Porto Alegre, v. 1, n. 2, p.24-28. 
LIU, Shimin et al. (2008). Optical absorption and emission properties of $\mathrm{Er}^{3+}$ doped mixed alkali borosilicate glasses. Optical Materials, n. 30, p.1393-1398.

NATALI, F. et al. (2015). Rare-earth mononitrides. Progress In Materials Science, n. 58, p.1316-1360, 2013. Disponível em: <www.elsevier.com/locate/pmatsci>. Acesso em: 16 mar. 2015.

$\mathrm{NI}$, Xin-long et al. (2015). Advances in the lanthanide metallosupramolecular chemistry of the cucurbit[n]urils. Coordination Chemistry Reviews, n. 287, p.89-113. Disponível em: <www.elsevier.com/locate/ccr>. Acesso em: 12 mar. 2015.

NISSAMUDEEN, K.M.; GOPCHANDRAN, K.G. (2015). $\mathrm{Y}_{2} \mathrm{O}_{3}: \mathrm{Eu}^{3+}$ based nanophosphors with higher oscillator strength through lithium incorporation and indirect oxidation. Journal Of Alloys And Compounds, n. 490, p.399-406, 2010. Disponível em: <www.elsevier.com/locate/jallcom>. Acesso em: 06 mar. 2015.

PEDROSA, A. M. G. et al. (2004). Propriedades espectroscópicas e parâmetros ópticos-eletrônicos dos complexos $\mathrm{Ln}\left(\mathrm{NO}_{3}\right)_{3} .2 \mathrm{DTPO} .4 \mathrm{H}_{2} \mathrm{O}(\mathrm{Ln}=\mathrm{Nd}$ e $\mathrm{Er}$ ). Quimica Nova, 27(3), p.367-370.

SEREDIN, V. V.; DAI, S. (2012). Coal deposits as potential alternative sources for lanthanides and yttrium. International Journal Of Coal Geology, n. 94, p.67-93.

Silva, A. G. et al. Parâmetros de Ligação e Avaliação de Intensidades Espectrais para Cloretos Hidratados de Lantanídeos.360 Cong. Assoc. Bras. Quím., 1996.

SOM, T.; KARMAKAR, B. (2011). Nephelauxetic effect of low phonon antimony oxide glass in absorption and photoluminescence of rare-earth ions. Spectrochimica Acta Part A: Molecular and Biomolecular Spectroscopy, n. 79, p.1766-1782.

SUCHOCKI, A.; BIERNACKI, S. W.; GRINBERG, M. (2007). Nephelauxetic effect in high-pressure luminescence of transition-metal ion dopants. Journal of Luminescence, n. 125, p.266-270. Disponível em: <www.elsevier.com/locate/jlumin>. Acesso em: 16 mar. 2015. 